\title{
AS BASES NEURAIS DA LINGUAGEM E O HEMISFÉRIO DIREITO NA CONSTRUÇÃO DE SENTIDOS
}

\author{
THE NEURAL BASES OF LANGUAGE AND THE RIGHT \\ HEMISPHERE IN THE CONSTRUCTION OFMEANING
}

\author{
Lucilene Bender de SOUSA ${ }^{1}$
}

Fernanda SCHNEIDER ${ }^{2}$

\begin{abstract}
Resumo: Os avanços teóricos e metodológicos decorrentes da neurociência cognitiva têm promovido importantes mudanças na forma como investigamos e concebemos a linguagem. Pesquisas têm cada vez mais utilizado técnicas, como a neuroimagem, em estudos multidisciplinares, com o objetivo de melhor compreender o processamento da linguagem no cérebro. Nesse contexto, este artigo apresenta uma revisão assistemática de importantes estudos sobre as bases neurais do processamento da linguagem e sobre as contribuições do hemisfério direito (HD) para o processamento discursivo e pragmático. Discutem-se alguns dos trabalhos mais relevantes e suas principais contribuições teóricas sobre a temática. Os resultados dos estudos analisados indicam que a linguagem não tem sua especialização em uma única região cerebral, mas envolve conexões entre diversas regiões dos dois hemisférios cerebrais. Além disso, parece haver um consenso quanto ao importante papel do HD na integração de informações semânticas, nos processos de elaboração de inferência e em atividades cognitivas complexas ligadas ao processamento pragmático-discursivo.
\end{abstract}

Palavras-chave: Linguagem. Processamento discursivo. Processamento pragmático. Hemisférios cerebrais. Hemisfério direito. Neuroimagem.

\begin{abstract}
The theoretical and methodological advances resulted from cognitive neuroscience have promoted important changes in the way that we investigate and conceive the language. Researches have used more techniques as neuroimaging in multidisciplinary studies to understand language processing in the brain. In this context, this article presents a nonsystematic review of important studies on the neural basis of language processing and about the contributions of the right hemisphere $(\mathrm{RH})$ to discursive and pragmatic processing. It discusses some of the most relevant studies and their main theoretical contributions on the topic. The results of the analyzed studies indicate that language does not have its specialization in a unique brain area but involves connections between many regions of both brain hemispheres. Moreover, it seems to exist a consensus about the relevant role of the $\mathrm{RH}$ in semantic data integration, inference processes, and complex cognitive tasks related to pragmatic-discursive processes.
\end{abstract}

Keywords: Language. Discourse processing. Pragmatic processing. Cerebral hemispheres. Right hemisphere. Neuroimaging.

\footnotetext{
1 Instituto Federal do Rio Grande do Sul (IFRS), Farroupilha, Rio Grande do Sul, Brasil; | ucilene.sousa@farroupilha.ifrs.edu.br; https://orcid.org/0000-0002-3833-5987
}

2 Instituto Federal do Rio Grande do Sul (IFRS), Ibiruba, Rio Grande do Sul, Brasil; fernanda.schneider@ibiruba.ifrs.edu.br; https://orcid.org/0000-0003-4412-8711 
- | As bases neurais da linguagem e o hemisfério direito na construção de sentidos

\section{Introdução}

A partir dos estudos das neurociências, surge o conceito de dominância ou de especialização cerebral, isto é, as diferenças de funções entre os dois hemisférios. Foi Paul Broca que, em 1861, realizou a primeira demonstração científica de assimetria funcional entre os hemisférios. O cientista propôs que uma perda na faculdade da linguagem articulada estava ligada a uma LHE (lesão no hemisfério esquerdo) (BELIN; FAURE; MAYER, 2008; HUTSLER; GALUSKE, 2003; KRISTENSEN; ALMEIDA; GOMES, 2001). Sua teoria estava baseada no paciente Leborgne (BROCA, 1861) que, por ter dificuldades no discurso, conseguia emitir apenas a monossílaba "tan".

Alguns meses depois, Broca conheceu um segundo paciente, Lelong, que também apresentou um discurso reduzido decorrente de um acidente vascular cerebral time postonset $^{3}$ de 1 (um) ano. O paciente de 84 anos conseguia dizer apenas cinco palavras: oui (sim), non (não), tois (uma pronúncia de trois (três), toujours (sempre) e Lelo (uma pronúncia de seu próprio nome). Na autópsia em Lelong, também foi encontrada lesão em região próxima da região do lobo frontal lateral do primeiro paciente - "Tan", e Broca relatou à Sociedade Anatômica como um importante caso, apontando, assim, para a localização da fala nessa área (DRONKERS et al., 2007). Cabe ressaltar que é atribuído a esse momento o início da neurolinguística ${ }^{4}$. Desde então, e mais especificamente nas últimas décadas, muitos estudos sobre dominância cerebral foram conduzidos, com destaque para um maior avanço metodológico e teórico.

Nesse sentido, Obler et al. (2010) apontam para o fato de que, embora pesquisas tenham identificado regiões corticais perisilvianas no hemisfério esquerdo como cruciais para a linguagem, o advento das técnicas de imagem cerebral permitiu a observação in vivo de regiões cerebrais implicadas no processamento da linguagem fora dessas áreas, no hemisfério esquerdo (HE), especialmente nas áreas anteriores, bem como demonstraram a importante participação do hemisfério direito (HD). Assim, tem crescido o foco na investigação sobre o papel dos hemisférios cerebrais e a colaboração funcional entre eles (BELIN; FAURE; MAYER, 2008; FRANKLIN et al., 2010; QI; LEGAULT, 2020).

Essas descobertas vão além, pois os avanços teóricos na pesquisa da linguagem somados à disponibilidade de técnicas experimentais de alta resolução na neurociência

3 Tempo após o AVC.

4 Obler e Caplan (1990) apresentam uma introdução aos estudos da Neurolinguística e da Afasiologia Linguística - dois campos interdisciplinares e emergentes na época da publicação da obra. 
cognitiva $^{5}$ estão mudando profundamente a forma como investigamos (GERNSBACHER; KASCHAK, 2003a; POEPPEL et al., 2012). Consequentemente, concebemos de modo diferente a forma como o cérebro processa a linguagem. Nessa perspectiva, quais seriam, então, as bases neurais da linguagem? De acordo com pesquisas realizadas nos últimos anos, o que é sugerido em relação à contribuição do HD para a linguagem? Visando responder a essas questões, realizamos uma revisão assistemática da literatura na qual elegemos estudos cujos resultados trazem importantes contribuições para a discussão da temática em foco. Primeiramente, apresentamos um quadro-resumo com as áreas de Brodmann apontadas como sendo as que mais diretamente estariam envolvidas na linguagem. Posteriormente, procuramos sintetizar as contribuições do HD para a construção dos sentidos, mais especificamente, para o processamento discursivo e pragmático.

\section{As bases neurais do processamento da linguagem no cérebro: ênfase no discurso}

Compreendemos, a partir dos estudos realizados nas últimas décadas, que as lesões no HE, mais especificamente em áreas como as estudadas por Broca (1861) e Wernicke (1994), podem afetar a linguagem devido a uma obstrução ou ruptura do vaso sanguíneo responsável por nutrir parte desse hemisfério cerebral (KANDEL; SCHWARTZ; JESSELL, 2003), e podem ter como consequência distúrbios da linguagem como, por exemplo, a afasia. A afasia, frequente em indivíduos que sofrem AVC (Acidente Vascular Cerebral), pode surgir decorrente de lesão focal adquirida no Sistema Nervoso Central (MORATO, 2012). Pode ser definida como uma alteração no conteúdo, na forma e no uso da linguagem e de seus processos cognitivos subjacentes, tais como, percepção e memória. De acordo com Chapey (1994) e Ortiz (2010), essa alteração é caracterizada por redução e disfunção, manifestadas em graus variados, de acometimento tanto na modalidade expressiva quanto na receptiva (compreensão, produção, leitura e escrita).

Nesse contexto, o estudo das lesões cerebrais e o olhar da neurociência da linguagem para a fisiologia cerebral favorecem a construção e a compreensão de muitos aspectos para a pesquisa linguística (FRANÇA, 2015). As áreas como a Psicolinguística Experimental, a Neurociência Cognitiva e a Neuropsicolinguística estabelecem-se "com o passo certo e com a granularidade de detalhes necessária em direção à caracterização do processamento da linguagem no cérebro" (FRANÇA, 2015, p. 172). Desse modo,

\footnotetext{
5 A neurociência cognitiva é um campo científico que surge da convergência da neurociência e da psicologia cognitiva. Aborda o estudo da função cerebral de uma perspectiva multidisciplinar, servindo de base para diferentes níveis de análise (REDOLAR-RIPOLL, 2014).
} 
- | As bases neurais da linguagem e o hemisfério direito na construção de sentidos

compreender como as funções da linguagem estão organizadas no cérebro trata-se de uma questão crítica e minuciosa (TREMBLAY; DICK, 2016). Com base em um estudo realizado por Price (2012), podemos afirmar que, nos últimos 20 anos, cresceu consideravelmente o número de pesquisas que abordam as bases neurais do processamento da linguagem. Assim, hoje, temos a clareza, por exemplo, de que tanto a produção oral quanto a compreensão dependem da ativação simultânea de múltiplas áreas do cérebro. Nesse sentido, Poeppel et al. (2012) apresentam duas "falhas" do clássico modelo das áreas consideradas cruciais para a linguagem. A primeira seria evidenciada a partir da relação deficits e local de lesão cerebral, ou seja, uma lesão na região de Broca não coincide necessariamente com a afasia de Broca e, da mesma forma, apresentar a afasia de Broca não significa ter uma lesão, especificamente, na região de Broca (POEPPEL et al., 2012). Sendo assim, os modelos atuais - que incorporam regiões adicionais a estes modelos apresentam-se melhores para a caracterização dos dados neuropsicológicos.

O segundo aspecto abordado por Poeppel et al. (2012) refere-se à restrição às poucas regiões cerebrais que compõem o modelo clássico das áreas da linguagem, o qual subestima, dramaticamente, o número e a distribuição das regiões do cérebro agora conhecidas como tendo papéis fundamentais na compreensão (ARDILA; BERNAL; ROSSELLI, 2014, 2016a; PRICE, 2012) e na produção (ARDILA; BERNAL; ROSSELLI, 2016a; BERNAL; ARDILA; ROSSELLI, 2016; INDEFREY, 2004) da linguagem. Além do papel do hemisfério direito (não dominante), antes desconsiderado (POEPPEL et al., 2012), outras regiões extra-sylvianas lateralmente esquerdas, como o giro temporal médio ou o lobo temporal anterior superior, são agora conhecidas como essenciais (NARAIN et al., 2003), assim como estudos evidenciam a participação de áreas subcorticais (KOTZ; SCHWARTZE, 2010).

No final do século XX, surgiu um novo modelo de estudo e de organização cerebral da linguagem: o modelo funcional (ARDILA; BERNAL; ROSSELLI, 2016b). As técnicas de neuroimagem, em especial, a ressonância magnética funcional (fMRI, do inglês Functional Magnetic Ressonance Imaging), permitiram avançar significativamente nesse sentido. A partir disso, estudos meta-analíticos (ARDILA; BERNAL; ROSSELLI, 2014; ROSSELLI; ARDILA; BERNAL, 2015) analisaram as áreas de Brodmann que, potencialmente, parecem participar tanto da produção (AB44, AB46) quanto da compreensão da linguagem, além de investigar o papel da ínsula (AB13) nos processos linguísticos. Nesses estudos, ressaltase que a ativação simultânea de várias áreas cerebrais em uma tarefa sugere que essas áreas fazem parte de um circuito cerebral relacionado para a função e domínio selecionados - o critério de filtro, por exemplo, a linguagem. Desse modo, se duas ou mais áreas se ativam durante a realização de uma tarefa linguística, significa que essas áreas se encontram interconectadas e participam de um circuito comum. 
Ardila, Bernal e Rosselli (2016a) observaram que a maioria das pesquisas inclui o primeiroe, algumas vezes, o segundogirotemporal esquerdo, que, em geral, correspondem às áreas $A B 21$ e $A B 22$, sendo também incluídas $A B 39$ e $A B 40$. No estudo realizado (ARDILA; BERNAL; ROSSELLI, 2016a), os pesquisadores analisaram o papel de AB20, AB37, AB38 e AB39 e seus conglomerados. Assim, perceberam que, em conjunto, os dados apontam para o fato de que essas áreas têm uma participação parcial na linguagem, podendo ser consideradas áreas de "associação linguística" (ARDILA; BERNAL; ROSSELLI, 2016a). Elas não podem ser consideradas áreas centrais, mas AB20, AB37, AB38 e AB39 apresentam certa participação nos processos linguísticos. As áreas AB37 (reconhecimento visual e funções semânticas), AB38 (contribui em vários circuitos cerebrais que fundamentam processos linguísticos relacionados à compreensão e à produção), com conglomerado de ativação com AB13 e AB22, no HE, e com AB7 e AB21 no HD - estão relacionados ao processamento da fala.

Nos últimos anos, surgiu um grande interesse em reanalisar especificamente as funções das subáreas de Broca. Assim, destacam-se os estudos que analisaram a AB44 (ARDILA et al., 2017; BERNAL; ARDILA; ROSSELLI, 2016) e a AB46 (ARDILA; BERNAL; ROSSELLI, 2016b). De acordo com esses achados, um conglomerado significativo inclui as áreas AB6, AB44, AB45, AB46 e AB47 no HE, o que implica, de acordo com os autores, dizer que o sistema frontal na sua totalidade participa da produção da linguagem (ARDILA; BERNAL; ROSSELLI, 2016b). Por fim, apresentam a ínsula, AB13, salientando que, durante muito tempo, aceitou-se que ela teria uma participação direta com a linguagem; lesões na ínsula podem resultar em alterações da linguagem, entre elas, a afasia de Broca, a afasia de condução e a afasia de Wernicke, mutismo e apraxia. Entretanto, Ardila, Bernale e Rosselli (2016b) argumentam que a ínsula não somente tem participação na linguagem, como sua participação é central. Assim, os conglomerados ativados nas conexões da ínsula, além de participação das áreas de produção (como a área de Broca) e compreensão (como a área de Wernicke), também se relacionam com a AB9 (processamento linguístico complexo) e AB37 (associação léxico-semântica).

Estudos envolvendo a produção de narrativas com neuroimagem (MAR, 2004) convergem no que se refere, ainda, à ativação do giro frontal (AB6, AB8, AB9 e AB10), das áreas próximas da região temporoparietal esquerda (BA22), lado direito (AB39/ AB40) e cingulado anterior (AB23, AB31). A Figura 1 apresenta as áreas de Brodmann e, mais especificamente, são destacadas as áreas que classicamente contribuiriam, mais diretamente, para a produção e a compreensão da linguagem.

A área frontal da linguagem (Fig. 1), ou complexo de Broca (ARDILA et al., 2017; ARDILA; BERNAL; ROSSELLI, 2016a), compreende a produção da linguagem e da 
- | As bases neurais da linguagem e o hemisfério direito na construção de sentidos

gramática - AB44, AB45, AB46 e AB47; e, também, parcialmente, inclui $A B 6$ e estendese, subcorticalmente, para os gânglios da base. A área de linguagem posterior abrange a recepção da linguagem e compreensão, o sistema léxico semântico - inclui o núcleo da área de Wernicke - AB21, $A B 22, A B 41, A B 42$ - e uma extensão da área de Wernicke, que pode ser observada nas áreas pontilhadas (Fig. 1), incluindo-se AB20, AB37, AB38, AB39 e AB40. A partir da revisão dos estudos discorridos, apresentamos, no Quadro 1, as áreas corticais.

Figura 1. Áreas de Brodmann e linguagem

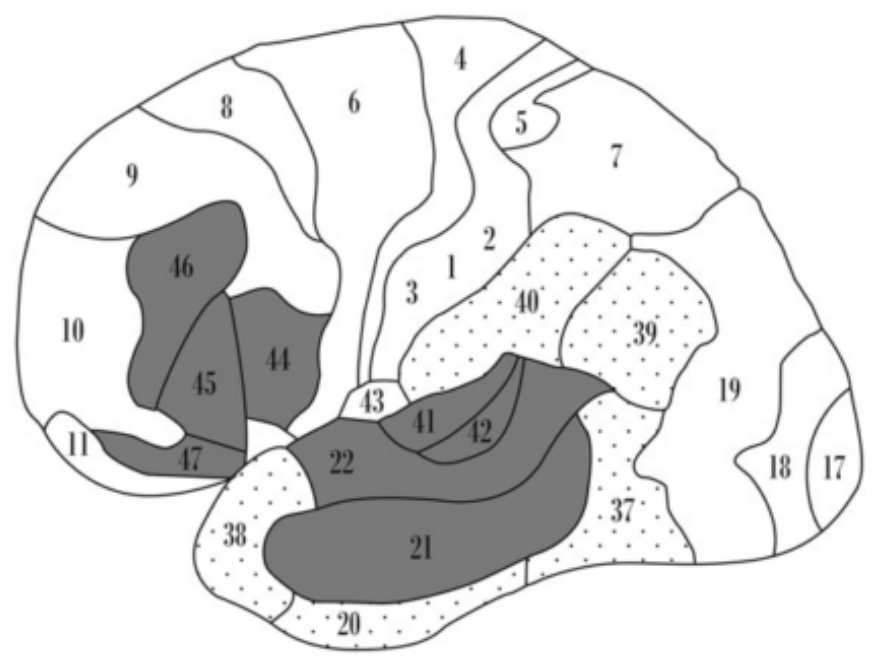

Fonte: Ardila, Bernal e Rosselli (2016a, p. 120)

Quadro 1. Áreas de Brodmann importantes para o processamento da linguagem

\begin{tabular}{|l|l|}
\hline \multicolumn{1}{|c|}{ Area } & \multicolumn{1}{|c|}{$\mathrm{AB}$} \\
\hline Pares operculares, parte da área de Broca & $\mathrm{AB} 44$ \\
\hline Frontal medial córtex frontal medial dorsolateral & $\mathrm{AB} 46$ \\
\hline Giro fusiforme & $\mathrm{AB} \mathrm{37}$ \\
\hline Area temporopolar & $\mathrm{AB} \mathrm{38}$ \\
\hline Area temporopolar (HD) & $\mathrm{AB} \mathrm{39,40*}$ \\
\hline Giro temporal superior anterior & $\mathrm{AB} 22$ \\
\hline Córtex de associação somatossensorial & $\mathrm{AB} 7$ \\
\hline Cingulado posterior & $\mathrm{AB} 23,31$ \\
\hline Giro temporal médio & $\mathrm{AB} 21^{*}$ \\
\hline Pares triangulares área de Broca & $\mathrm{AB} 45$ \\
\hline Córtex pré-frontal dorsolateral & $\mathrm{AB} 46$ \\
\hline Orbital/ córtex frontal ventrolateral & $\mathrm{AB} 47$ \\
\hline Córtex frontal & $\mathrm{AB} 6,8,9,10$ \\
\hline Insula & $\mathrm{AB} 13$ \\
\hline
\end{tabular}

Legenda: $\mathrm{AB}=$ Área de Brodman. ${ }^{*} \mathrm{HD}$ : Hemisfério Direito.

Fonte: Elaboração própria 
Das áreas de Brodmann que estão mais diretamente implicadas, de acordo com a literatura, no processamento da linguagem (Quadro 1), Price (2012) acrescenta, ainda, como importantes para a linguagem, áreas como o putâmen - estrutura localizada na base da parte frontal do cérebro; o cerebelo - localizado na parte posterior do cérebro; e o tálamo - situado no diencéfalo (entre o córtex cerebral e o mesencéfalo).

Desse modo, sendo todas essas áreas fundamentais para a linguagem, o que acontece quando ocorre uma lesão no cérebro em alguma dessas importantes áreas? Podemos dizer que vários fatores estão implicados, mas, em alguns casos, as sequelas decorrentes de lesões pós AVC, por exemplo, resultam na dificuldade ou perda da linguagem. Nesse sentido, como inicialmente acreditava-se que o hemisfério esquerdo (HE) era quase exclusivamente o responsável pelo processamento da linguagem, muitos estudos acabaram centrando-se a partir dessa consideração. Entretanto, mais recentemente, as pesquisas buscaram investigar as contribuições do HD para a linguagem, tema abordado nas próximas seções do presente estudo, com enfoque no processo de compreensão discursiva e pragmática.

\section{Contribuições do HD para o processamento semântico e discursivo}

À medida que os estudos sobre cérebro e linguagem avançam, percebe-se a tendência a uma interpretação menos localizacionista e mais integradora da atividade cerebral. Evidências têm demonstrado que o processamento ocorre em redes paralelas em diversas áreas e que os hemisférios trabalham de forma colaborativa. Com relação ao aspecto semântico, segundo Kahlaoui, Scherer e Joanette (2008), um conjunto complexo de comunicações e interações hemisféricas parece estar envolvido, sendo cada hemisfério responsável por diferentes tipos de conhecimentos e relações semânticas. A Figura 2 apresenta algumas das regiões cerebrais que serão mencionadas nesta seção cujo foco é o papel do HD nos processos de integração e inferência envolvidos na compreensão do discurso. 
- | As bases neurais da linguagem e o hemisfério direito na construção de sentidos

Figura 2. Ilustração de algumas das regiões cerebrais relacionadas ao processamento de sentidos

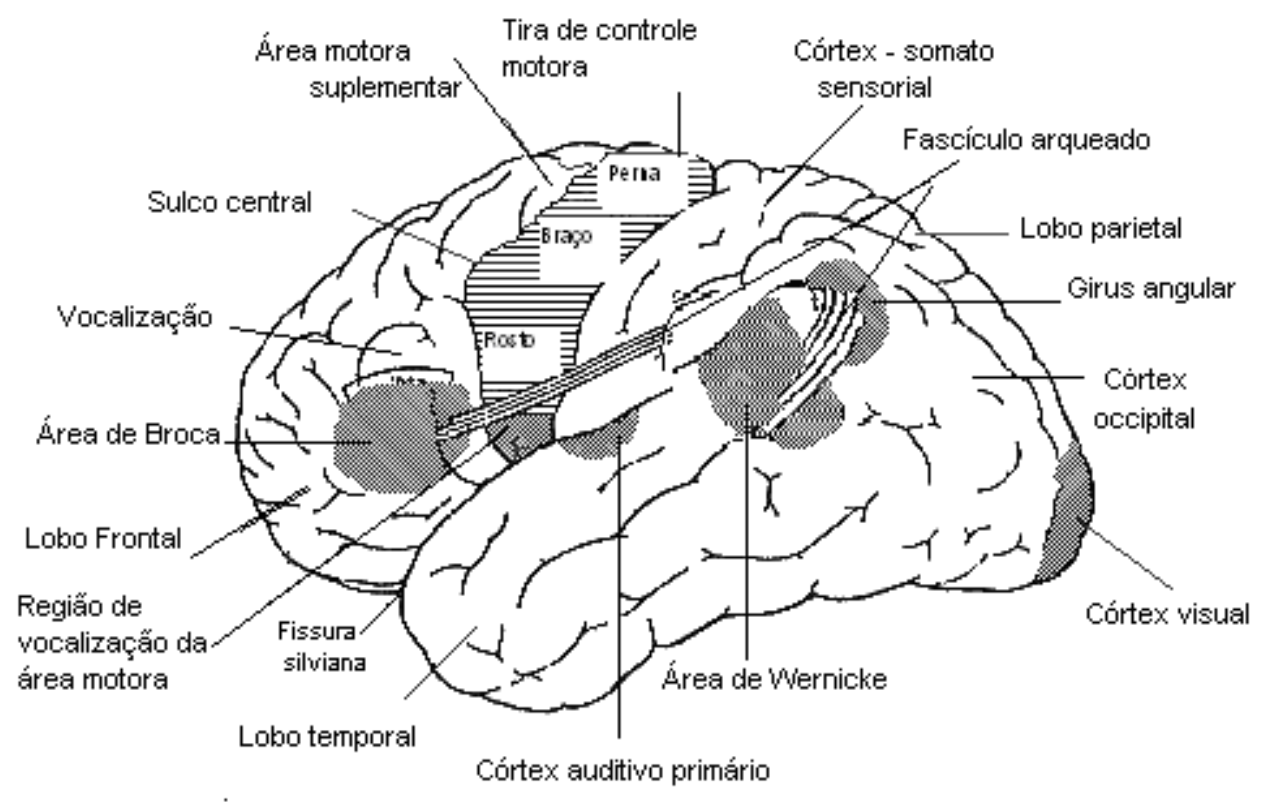

Fonte: Traduzido e adaptado de Biblioteca Pleyades (2019).

O processamento semântico, conforme Jung-Beeman (2005) depende basicamente de três etapas: ativação, integração e seleção. Por meio da ativação, o cérebro acessa a representação semântica que está distribuída em rede. Essa etapa parece ocorrer bilateralmente nas áreas de Wernicke (Figura 2), principalmente no giro temporal posterior médio e superior. Os hemisférios são sensíveis às diferenças de contextos, ou seja, segundo o autor, o HE ativa sentidos dominantes, literais e contextualmente relevantes, enquanto o HD mantém ativação fraca e difusa de um campo semântico mais amplo, por isso torna-se mais sensível a relações semânticas distantes. Esse tipo de ativação realizada pelo HD foi denominada por Beeman (1998) de "coarse semantic coding", ou seja, codificação semântica ampla. Dessa forma, ele propõe que a diferença entre o processamento semântico dos dois hemisférios é de ordem qualitativa, sendo que o HE ativa pequenos campos semânticos (fine semantic coding) que estão forte e diretamente ligados; enquanto o HD ativa grandes campos semânticos que se sobrepõem e mantêm a ativação por maior tempo, embora suas representações sejam mais fracas.

O processo de integração é essencial para a busca de coerência entre as diversas ativações semânticas, uma vez que, através dele, associam-se as diferentes redes ou campos semânticos, o que é fundamental para a compreensão de frases e textos. A integração parece envolver as seguintes áreas de ambos os hemisférios: giro temporal anterior superior, sulco superior temporal, giro temporal médio e polo temporal (JUNG- 
BEEMAN, 2005). A seleção do sentido apropriado, por sua vez, envolve a escolha dentre as diversas possibilidades de sentido do que é relevante para o contexto. A principal área ativada na seleção é o giro inferior frontal esquerdo, havendo evidências também da contribuição da mesma área no HD. As três etapas do processamento semântico, propostas por Beeman (1998), ocorrem de forma altamente integrada.

No que se refere ao processamento do discurso, é importante lembrar que sua demanda cognitiva é maior se comparada ao processamento semântico no nível da palavra, distinguindo-se basicamente pela necessidade de maior integração de informações na busca de coerência e de coesão textual. Segundo Newman, Just e Mason (2004), o HD desempenha um papel importante para essa tarefa, além de participar da elaboração de inferências. O HD parece ser ativado à medida que aumenta a complexidade das tarefas, o que se confirma pelos estudos a serem apresentados a seguir, tanto no processamento discursivo quanto no pragmático.

Nesse sentido, na última década, temos maiores evidências de que a produção oral - assim como a compreensão - depende da participação simultânea de múltiplas áreas do cérebro, nos dois hemisférios cerebrais. Gernsbacher e Kaschak (2003b), ao realizarem uma revisão de pesquisas em neuroimagem sobre a produção e compreensão da linguagem, afirmaram que o processamento envolvido no uso da linguagem ocorre em regiões difusas do cérebro. Os autores identificaram as regiões temporais e frontais do HD como áreas importantes para os aspectos integrativos, por exemplo, no processamento narrativo, enfatizando a hipótese de que a ativação observada nessas áreas específicas ao processamento do discurso pode ser uma função dos processos de memória.

MasoneJust(2006)apontam cincoredes paralelas especializadasnoprocessamento discursivo: 1) rede de processamento abrangente (temporal médio e superior direito); 2) rede de monitoramento de coerência (pré-frontal dorsolateral bilateral); 3) rede de integração textual (frontal inferior esquerdo e temporal anterior esquerdo); 4) rede para interpretar o protagonista ou perspectiva do agente (frontal medial/posterior bilateral, temporal/parietal direito); e 5) rede de imagem espacial (esquerdo dominante, sulco intraparietal bilateral).

No estudo citado, os autores (MASON; JUST, 2006) exploram os trabalhos que indicam a existência dessas redes assim como a teorização a respeito de cada uma delas. Eles deixam claro que não há certeza sobre a realidade dessas redes e sua localização anatômica, sendo necessários mais estudos para sua comprovação. Outro ponto relevante é sua proposta de localização no cérebro do modelo situacional do texto, segundo a qual a representação do texto é difusa e distribuída por muitas áreas de acordo com a natureza 
- | As bases neurais da linguagem e o hemisfério direito na construção de sentidos

da informação, por exemplo, a informação espacial é representada na região parietal do HD, já a informação emocional na amígdala/córtex frontal medial (MASON; JUST, 2006); por isso, sugerem que desenvolver um grupo de redes de processamento do discurso exigiria tantas e tão variadas redes quanto os textos existentes.

Segundo Scherer (2009), dados de neuroimagem evidenciam a atividade colaborativa e complementar entre os dois hemisférios, em que o HD, especialmente suas regiões temporais mediais, executa a integração semântica em nível discursivo, proporcionando a coerência global, e o HE executa o processo de integração local, no nível inter e intrassentencial, sendo o hipocampo e o precúneo as principais regiões ativadas nos processos de memória necessários para essa integração. No entanto, não há consenso sobre os processos cognitivos específicos envolvidos na coerência global (MAR, 2004; KURCZEK; DUFF, 2012; MARINI et al., 2011).

Ainda no que se refere à coesão e coerência textual, com foco no papel da atenção e das funções executivas ${ }^{6}$ (FEs), Barker, Young e Robinson (2017) investigaram, utilizando a técnica de $\mathrm{FMRI}$, participantes que sofreram $\mathrm{AVC}$, mas que não foram diagnosticados como afásicos. Foram recrutados 6 participantes com LHE (lesão no hemisfério esquerdo) (média de idade 66.5), 6 participantes com LHD (lesão no hemisfério direito), (média de idade 62), 4 participantes com lesão bilateral e 21 participantes no grupo controle. Os resultados mostraram déficits no processamento da coesão e da coerência textuais no grupo experimental, em relação ao grupo controle, principalmente nos participantes com lesão no HD. No grupo clínico, um melhor desempenho em tarefas de atenção, por exemplo, foi relacionado a menos repetições proposicionais e a menores erros de coerência global. Os resultados desse estudo são, de acordo com os autores, os primeiros a indicar que os déficits na coesão e coerência podem ocorrer após AVC, sem a presença de afasia. Além disso, os resultados encontrados evidenciam o papel das funções executivas e da atenção no discurso.

Um dos processos discursivos que mais interessa aos pesquisadores, além da coesão e da coerência mencionadas acima, é a geração de inferência, na qual o HD parece estar especialmente envolvido. Virtue et al. (2006) investigaram a atividade neural na realização de inferências durante a compreensão de histórias. Os autores apontaram para uma complexa rede de processos envolvidos na inferência, em que participam o giro frontal inferior (GFI) bilateral na etapa de mapeamento cognitivo, seleção e recuperação

6 As funções executivas tornam possível organizar mentalmente as ideias; reservar um tempo para pensar antes de agir, antecipar desafios; resistir às tentações e ficar focado (DIAMOND, 2013). Ou seja, elas constituem um conjunto de habilidades que são essenciais para o controle sobre ações, pensamentos e emoções. As três dimensões fundamentais das FEs são: memória de trabalho, controle inibitório e flexibilidade cognitiva. 
semântica da informação inferencial; e o giro temporal superior (GTS) na etapa de integração da inferência semântica; sendo que o GTS direito demonstrou ser inicialmente mais sensível a conexões causais do que o esquerdo, envolvendo-se, portanto, antes no processo inferencial. Além da participação no processo de inferência, os autores estendem a participação das áreas citadas à construção da representação da coerência textual. $O$ GTS direito aumenta sua atividade quando a inferência é relacionada a eventos implícitos, enquanto o GTS esquerdo é ativado quando ocorre uma ruptura de coerência. Ambos os hemisférios participam de forma colaborativa no processamento inferencial, porém os autores fazem a seguinte distinção: o HD é mais sensível a conceitos relacionados a inferências preditivas, inferências anafóricas com múltiplos antecedentes, e inferências ponte e preditivas em textos fracamente restritivos, ao passo que o HE mostra-se mais sensível a conceitos relacionados à inferência ponte, inferências anafóricas com um antecedente, inferência ponte e preditiva em textos fortemente restritivos.

Outra pesquisa relevante sobre esse tema foi desenvolvida por Silagi et al. (2018) que compararam a geração de inferência em leitores com LHD e LHE. Eles verificaram que os dois grupos apresentavam dificuldades em inferências. Porém, os participantes com LHD tiveram desempenho inferior aos com LHE nas questões que requeriam a produção de inferências mais complexas como as lógicas e pragmáticas. Além disso, os resultados corroboram o que já foi apontado no estudo de Barker, Young e Robinson (2017). Os participantes com LHD mostraram correlação significativa entre a habilidade de produção de inferências lógicas e a atenção, a memória, as funções executivas e as funções visuoespaciais. Já em participantes com LHE, os resultados mostraram correlação entre inferências pragmáticas, atenção e habilidades visuoespaciais.

Há também evidência do envolvimento de duas regiões de massa branca (fascículo arqueado - Fig. 2 e fascículo longitudinal inferior), em ambos os hemisférios, com a compreensão discursiva. Os resultados do experimento conduzido por Horowitz-Kraus e colegas (2014) revelaram forte correlação entre o desempenho na compreensão discursiva e o fascículo arqueado direito. Interessante observar que a correlação com o fascículo arqueado direito foi superior à do esquerdo e também a do fascículo longitudinal inferior esquerdo. Os autores, inclusive, interpretam o resultado com a hipótese de que regiões do lobo frontal direito, conectadas às áreas posteriores dos lobos temporais e parietais, associadas à linguagem, compõem o circuito da compreensão leitora.

Por fim, outros estudos relevantes para a compreensão do papel do HD no processamento do discurso foram desenvolvidos e cabem ser citados. St George et al. (1999) pesquisaram sobre a ativação cerebral em textos sem referente/título versus textos em que um título é dado no início da leitura; Caplan, Dapretto e Mazziotta (2000) 
- | As bases neurais da linguagem e o hemisfério direito na construção de sentidos

examinaram a manutenção da lógica e do tópico na compreensão oral; Ferstl e Von Cramon (2002) contrastaram aspectos emocionais e aspectos temporais da construção do modelo situacional; Tomitch et al. (2004) investigaram a diferença de processamento entre os dois hemisférios durante integração textual; Johns, Tooley e Traxler (2008) realizaram uma ampla revisão de estudos que abarcavam o papel do HD no processamento discursivo, inferência, humor e linguagem não-literal; Vigneau et al. (2011), cuja meta-análise examinou a contribuição do HD no processamento fonológico, léxico-semântico e sentencial. Todos demonstram a relevante participação do HD, especialmente nas tarefas de integração semântica e inferência, bem como seu importante papel no processamento da informação contextual, tema que será abordado na próxima seção.

A partir dos estudos mencionados, percebemos que, apesar de serem sugeridas, durante anos, áreas cruciais para a linguagem, hoje, parece predominar a acepção de que o discurso está associado a redes e não simplesmente a áreas cerebrais - como sugerido por Ardila et al. (2017) ao investigarem a produção oral. Essa definição justifica as novas abordagens e conhecimentos advindos de uma nova neurobiologia da linguagem ${ }^{7}$ (POEPPEL et al., 2012) que se constitui como uma área de investigação promissora ao integrar a neurociência e a linguística.

\section{Contribuições do HD para o processamento pragmático}

Os aspectos pragmáticos da linguagem relacionam-se diretamente ao seu uso, o qual está vinculado ao social, às convenções, à figuratividade e à percepção da intencionalidade comunicativa. Dessa forma, esse processamento exige um alto nível de interpretação da situação/contexto e do ato enunciativo entre os interlocutores. Ao abordar o processamento pragmático, centramo-nos na compreensão da metáfora, da ironia e da Teoria da Mente (em inglês, Theory of Mind - ToM) ${ }^{8}$, exemplos altamente ilustrativos da linguagem no contexto da pragmática.

7 A neurobiologia da linguagem investiga as bases neurais da linguagem no cérebro. É um campo de pesquisa que teve muitos progressos nas últimas décadas. Os avanços ocorreram, principalmente, pela integração das abordagens neurobiológicas com o entendimento mais estabelecido da linguagem dentro da psicologia cognitiva, ciência da computação e linguística (HICKOK; SMALL, 2016).

8 A Teoria da Mente (ToM) diz respeito à habilidade de atribuir intenções, sentimentos, desejos e crenças e, baseado neles, predizer o comportamento das pessoas. O termo foi criado por Premack e Wooddruff (1978) que realizaram uma pesquisa sobre sistema de inferências em chimpanzés. Mais adiante, Wimmer e Perner (1983) utilizaram o termo e investigaram em que momento do desenvolvimento do ser humano surgia a então denominada "teoria da mente". 
No que se refere à metáfora, vários estudos têm sido realizados, porém, há divergências quanto à especialização do HD no seu processamento. Giora et al. (2004) observaram que pacientes com LHD tiveram desempenho significativamente inferior aos pacientes com LHE na compreensão do sarcasmo (frases irônicas). Já na tarefa com metáforas convencionais, os pesquisadores verificaram que pacientes com LHE apresentaram desempenho significativamente inferior aos participantes normais enquanto nos pacientes com LHD essa diferença não foi significativa. Esses resultados confirmaram a sua Hipótese do Grau de Saliência - graded salience hypothesis (GIORA, 1997), segundo a qual o processamento de sentidos salientes, convencionais como metáforas do tipo "coração partido", é realizado predominantemente pelo HE; enquanto o processamento de sentidos não salientes como a ironia é realizado predominantemente pelo HD. A autora propõe, portanto, que o principal fator determinante para o envolvimento do HD não é a figuratividade, sentido literal ou figurativo, mas sim a convencionalidade/novidade, o quão novo é aquele sentido para o leitor ou o ouvinte.

No entanto, há estudos cujos resultados contrariam essa hipótese. No experimento realizado por Arzouan, Goldstein e Faust (2007) por meio de EEG (Eletroencefalografia), os resultados apontaram para o envolvimento de ambos os hemisférios na compreensão de metáforas. No HD, observaram ativação nas regiões temporal e superior frontal, áreas semelhantes a essas foram identificadas no HE durante o processamento de metáforas novas. O HE mostrou maior ativação durante a compreensão de metáforas convencionais, com participação em menor grau também do HD. Os autores observaram ainda diferença no tempo de ativação: o HD era ativado em estágios recentes e médios, com gradual queda no estágio posterior, enquanto o HE era ativado apenas na fase tardia. Arzouan e colegas (2011) também constataram a participação de ambos os hemisférios no processamento tanto de metáforas convencionais quanto de metáforas novas.

Outro estudo relevante foi o conduzido por Schmidt e Seger (2009), por meio da técnica de $\mathrm{fMRI}$, no qual a familiaridade, a imageabilidade e o grau de dificuldade das frases foram controladas. Os resultados também confirmam a ativação do HD no processamento metafórico, porém eles concluíram que a imageabilidade sozinha pode não ser o ponto crucial para o recrutamento do HD, já que ativa ambos os hemisférios. Os demais fatores - familiaridade e dificuldade - fortemente relacionados, também se mostraram determinantes para a participação do HD. Os pesquisadores sugerem que outras especialidades do HD, como a ativação semântica ampla, funções da memória de trabalho e processos de integração podem estar ligados ao processamento metafórico. O estudo contribui no sentido de mostrar a importância do controle do estímulo e o impacto que fatores como o tipo de metáfora e o grau de complexidade podem ter sobre os resultados. 
- | As bases neurais da linguagem e o hemisfério direito na construção de sentidos

A meta-análise realizada por Yang (2014) mostra que complexidade contextual, convencionalidade e demanda textual influenciam no envolvimento do HD na compreensão da metáfora uma vez que modulam o efeito de figuratividade. Diaz e Eppes (2018), por sua vez, analisaram fatores que interferem no envolvimento do HD no processamento de metáforas. Eles propõem que o que esses fatores têm em comum não é apenas a figuratividade da linguagem utilizada, mas principalmente a demanda aumentada de integração.

A pesquisa do processamento de ironia também é um campo promissor dentro da pragmática, no entanto, com poucos estudos de neuroimagem. Eviatar e Just (2006) compararam o processamento de três grupos de frases-histórias diferentes: significado literal, metafórico convencional e irônico. Além de encontrar ativação nas áreas clássicas da linguagem, encontraram ativação diferenciada do HD, giro temporal médio e superior, durante a compreensão de ironias; e do HE, giro frontal inferior, na compreensão de metáforas convencionais. Os autores concluíram que o processamento da linguagem figurativa possivelmente ocorra pelo mesmo mecanismo pelo qual são processadas frases literais (áreas clássicas). Consideram que tanto metáforas quanto ironias são funções bilaterais, com assimetria na quantidade de ativação dos dois hemisférios, portanto, com variado grau de lateralização.

Shibata et al. (2010) investigaram os substratos neurais no processamento de ironias em frases ecoicas. Eles compararam a ativação cerebral na compreensão de frases em três condições: sentido irônico, sentido literal e sem sentido. Após subtrair as regiões ativadas no processamento do sentido literal e do sentido irônico, verificaram que as regiões préfrontal medial (BA10) e pré-central (BA6) do HD e sulco temporal superior (BA21) do HE foram ativadas apenas no processamento da ironia, confirmando a participação do HD nesse processo.

A meta-análise de Bohrn, Altmann e Jacobs (2012) comparou 23 estudos de neuroimagem no intuito de identificar os circuitos neurais associados ao processamento da linguagem figurativa e literal. Eles verificaram no processamento de metáforas e expressões idiomáticas um padrão de ativação específico no giro frontal inferior esquerdo. Já o processamento de ironias e sarcasmo exibiu ativação no córtex pré-frontal medial e ativação frontotemporal no HD. Assim como nos resultados de Eviatar e Just (2006), anteriormente citados, a análise sugere que o processamento da linguagem literal e figurada compartilham de uma rede bilateral de regiões frontotemporais com alguns componentes em comum e alguns componentes distintos. $\mathrm{O}$ estudo evidencia que o processamento de diferentes tipos de linguagem figurativa ocorre por meio de estratégias cognitivas distintas. 
A compreensão da metáfora parece envolver processos semânticos mais analíticos, já a compreensão da ironia requer processos associados à teoria da mente (ToM). Além da ToM, a pesquisa de Champagne-Lavau et al. (2018), com participantes que apresentavam LHD, revelou que o processamento da ironia também depende fortemente da habilidade de identificar informações contextuais relevantes. Os resultados mostraram que alguns participantes conseguiam compreender ironias e demonstravam maior sensibilidade às mudanças de informação contextual. Porém, um grupo menor de participantes não foi capaz de compreender as ironias e mostrou dificuldade em detectar as informações do contexto que eram relevantes para tal compreensão.

Parola et al. (2016) gestures, com o objetivo de oferecer uma avaliação multifocal das habilidades pragmáticas, realizaram um estudo em participantes com lesão no hemisfério direito (LHD). Os pesquisadores analisaram o desempenho de 17 participantes LHD e 17 controles saudáveis usando a Bateria de Avaliação para Comunicação (Assessment Battery for Communication de Bosco) (BOSCO et al., 2012), uma ferramenta clínica para avaliar uma ampla gama de fenômenos pragmáticos - tanto na compreensão quanto na produção. Os resultados sugerem que participantes com LHD têm dificuldade em compreender e produzir fenômenos pragmáticos de diferentes complexidades, de modo mais particular, os participantes parecem ter maiores prejuízos na modalidade não-verbal (gestos, expressões faciais).

Por fim, um dos tópicos diretamente relacionados com o processamento pragmático é a Teoria da Mente (ToM). Uma das pesquisas mais exponenciais na área foi desenvolvida por Saxe (2009). Ela define ToM como o mecanismo utilizado por alguém para inferir e raciocinar sobre o estado de mente das outras pessoas, de forma que possa compreender seus desejos, crenças e intenções. A rede ToM é incrivelmente robusta e fornece uma rara janela através do cérebro para a mente (SAXE, 2009). Segundo a autora, as regiões que a compõem são: a junção temporo-parietal bilateralmente, o córtex medial parietal e córtex pré-frontal medial. Uma das tarefas que pode recrutar a rede ToM é a leitura de narrativas. Saxe (2006) caracteriza essa leitura como um "verdadeiro milagre", pois partindo de um número restrito de frases, o leitor é capaz de perceber a mente humana, seus sentimentos, pensamentos, enganos e percepções. Em um de seus estudos, Saxe et al. (2006) demonstram que refletir sobre os pensamentos de personagens e atribuir traços de sua própria personalidade ativam parte da rede ToM, sub-regiões do precúneo medial e córtex medial pré-frontal. Regiões similares podem também estar envolvidas na memória episódica autobiográfica.

Há diversos estudos que buscaram identificar a interação das bases neurais da ToM com outros processos cognitivos como a coerência (FERSTL; VON CRAMON, 
- | As bases neurais da linguagem e o hemisfério direito na construção de sentidos

2002), julgamentos morais (YOUNG et al., 2007), controle executivo (CARLSON; MOSES; BRETON, 2002), pragmática e lesões cerebrais (CHAMPAGNE-LAVAU; JOANETTE, 2009), e compreensão de narrativas em autistas (MASON et al., 2008), compreensão leitora (ATKINSON et al., 2017; ZIV; SMADJA; ARAM, 2015) e leitura literária (KIDD; CASTANO, 2013).

Como podemos observar nos estudos supracitados, o processamento do discurso é realizado com maior ou menor participação do HD dependendo de fatores ligados à complexidade textual como a familiaridade, a convencionalidade, a modalidade e a dificuldade da linguagem utilizada. Os processos de compreensão que requerem maior integração entre as informações textuais e entre o texto-contexto-conhecimento de mundo dependem da ativação de uma rede neural mais ampla e demandam mais atenção, uso da memória e funções executivas. O HD era mais convencionalmente associado ao processamento pragmático envolvendo a linguagem figurada, porém, os estudos revisados mostram que a figuratividade, em si, não é o principal aspecto linguístico responsável pela ativação do HD. Assim, verificamos que apesar de o HD ter um importante papel no processamento discursivo e pragmático, ainda não há consenso sobre quais componentes linguísticos e cognitivos são decisivos para o seu recrutamento.

\section{Considerações finais}

No presente estudo, apresentamos evidências das bases neurais do processamento da linguagem, a partir da revisão de algumas das importantes pesquisas que abordam a temática, como as desenvolvidas por Poeppel et al. (2012), Mason e Just (2006) e Gajardo-Vidal et al. (2018). Além disso, a partir de estudos de neuroimagem (EVIATAR; JUST, 2006; MAR, 2004) e meta-analíticos (ARDILA; BERNAL; ROSSELLI, 2014; BERNAL; ARDILA; ROSSELLI, 2016), sintetizamos um espectro de possibilidades de participação do HD na construção de sentidos na linguagem. A partir da década de 90, a participação do HD tem sido considerada decisiva no processamento da linguagem, principalmente no que se refere ao nível discursivo e pragmático. As investigações dos diferentes níveis de processamento linguístico sugerem que quanto maior o grau de demanda semântica e/ou lexical, por exemplo, maior é a contribuição do HD (MALLOY-DINIZ; FUENTES; CONSENZA, 2013).

Nesse contexto, ressaltamos que, ao compreendermos o uso da língua de forma menos estrutural, menos centrada nas unidades mínimas, e mais global, centrado no texto e contexto, reconhecemos que o uso da língua para a comunicação, também denominado na literatura como a língua natural, envolve os dois hemisférios em graus e especializações 
diferentes, todavia, ambos com valor decisivo e complementar. Destacamos, ainda, o fato de que o papel do HD no processamento da linguagem por muito tempo foi considerado principalmente pragmático; no entanto, a partir do desenvolvimento de estudos como os apresentados nesta revisão (GIORA et al., 2004; ARZOUAN; GOLDSTEIN; FAUST, 2007) percebe-se que ambos os hemisférios integram-se tanto na compreensão discursiva quanto na pragmática.

Desse modo, muitas investigações foram desenvolvidas nos últimos anos sobre especializações cerebrais e apontaram aspectos importantes que contribuem para a compreensão do processamento da linguagem no cérebro. No entanto, a participação exata de cada um dos hemisférios cerebrais nesse processamento, como já mencionamos, ainda não foi totalmente evidenciada. Apesar das lacunas, grande progresso tem sido feito, principalmente devido ao avanço das técnicas de neuroimagem (por exemplo, a ressonância magnética por tensor de difusão - DTI, do inglês Diffusion Tensor Imaging - e fMRI). Essas técnicas poderão trazer ainda mais contribuições para a área da linguística na medida que abordarem diferentes aspectos do discurso, uma vez que, durante muito tempo, a maioria dos estudos envolvendo a linguagem ocorria no nível da palavra ou da sentença - e, ainda, muitos deles sem a participação e contribuição do linguista.

Sendo assim, reitera-se o fato de que as pesquisas com neuroimagem, integradas às comportamentais, ainda precisam avançar em muitos aspectos nos estudos linguísticos, considerando-se que a variabilidade semântica natural da língua sempre traz desafios para as neurociências. Além disso, fica evidente, a partir da análise das pesquisas apresentadas neste estudo, a grande influência que exercem o design experimental, o tipo de tarefa e a complexidade e variabilidade do estímulo linguístico nos resultados obtidos por estudos comportamentais e de neuroimagem, confirmando que a participação de linguistas em experimentos envolvendo a linguagem é fundamental para que se tenha maior controle e precisão dos estímulos e, consequentemente, resultados mais confiáveis.

\section{Referências}

ARDILA, A.; BERNAL, B.; ROSSELLI, M. Should broca's area include brodmann area 47?

Psicothema, v. 29, n. 1, p. 73-77, 2017.

ARDILA, A.; BERNAL, B.; ROSSELLI, M. How localized are language brain areas? A review of Broadmann areas involvement in oral language. Archives of Clinical Neuropsychology, v. 31, p. 112-122, 2016 a. 
- | As bases neurais da linguagem e o hemisfério direito na construção de sentidos

ARDILA, A.; BERNAL, B.; ROSSELLI, M. The language area of the brain: a functional reassessment. Revista de Neurolologia, v. 62, n. 3, p. 97-106, 2016 b.

ARDILA, A.; BERNAL, B.; ROSSELLI, M. The language area of the brain: a functional reassessment. Revista de Neurologia, v. 62, n. 3, p. 97-106, 2016c.

ARDILA, A.; BERNAL, B.; ROSSELLI, M. Participation of the insula in language revisited: a metaanalytic connectivity study. Journal of Neurolinguist, v. 29, p. 31 41, 2014.

ARZOUAN, Y.; GOLDSTEIN, A.; FAUST, M. Dynamics of hemispheric activity during metaphor comprehension: Electrophysiological measures. Neurolmage, v. 36, n. 1, p. 222-231, 2007.

ARZOUAN, Y.; SOLOMON, S.; FAUST, M.; GOLDSTEIN, A. Big words, halved brains and small worlds: Complex brain networks of figurative language comprehension. PloS one, v. 6, n. 4, p. e19345, 2011.

ATKINSON, L.; SLADE, L.; POWELL, D. Theory of mind in emerging reading comprehension: A longitudinal study of early indirect and direct effects. Journal of Experimental Child Psychology, v. 164, p. 225-238, 2017.

BARKER, M. S.; YOUNG, B.; ROBINSON, G. A. Cohesive and coherent connected speech deficits in mild stroke. Brain and Language, v. 168, n. 168, p. 23-36, 2017.

BEEMAN, M. Coarse semantic coding and discourse comprehension. In: BEEMAN, M.; CHIARELIO, C. (ed.). Right hemisphere language comprehension: Perspectives from cognitive neuroscience. Mahwah, NJ: Lawrence Erlbaum Associates Inc., 1998. p. 255-284.

BELIN, C.; FAURE, S.; MAYER, E. Hemispheric specialisation versus interhemispheric communication. Revue Neurologique, v. 164, n. 1, p. 48-53, 2008.

BERNAL, B.; ARDILA, A.; ROSSELLI, M. Broca's area network in language functions. A metaanalytic connectivity map based on analysis likelihood estimates. Frontiers in Psychology, v. 6, p. 660-687, 2016.

BIBLIOTECA PLEYADES. Disponível em: http://www.bibliotecapleyades.net/ciencia/ imagenes/psyco08_01.gif. Acesso em: 5 jun. 2019. 
BOHRN, I. C.; ALTMANN, U.; JACOBS, A. M. Looking at the brains behind figurative language - A quantitative meta-analysis of neuroimaging studies on metaphor, idiom, and irony processing. Neuropsychologia, v. 50, n. 11, p. 2669-2683, 2012.

BOSCO, F. M.; ANGELERI, R.; ZUFFRANIERI, M.; BARA, B. G.; SACCO, K. Assessment Battery for Communication: Development of two equivalent forms. Journal of Communication Disorders, v. 45, n. 4, p. 290-303, 2012.

BROCA, P. P. Perte de la parole: ramollissement chronique et destruction partielle du lobe antérieur gauche du cerveau. Bulletins de la Societe d'anthropologie, v. 2, p. 235$238,1861$.

BROCA, P. P. Localisations des fonctions cérébrales. Siège de la faculté du langage articulé. Bulletin de la Société, v. 4, p. 200-208, 1863.

CAPLAN, R.; DAPRETTO, M.; MAZZIOTTA, J. C. An fMRI study of discourse coherence. Neurolmage, v. 11, n. 55, p. S96, 2000.

CARLSON, S. M.; MOSES, L. J.; BRETON, C. How Specific is the Relation between Executive Function and Theory of Mind? Contributions of Inhibitory Control and Working Memory. Infant and Child Development, v. 11, n. 2, p. 73-92, 2002.

CHAMPAGNE-LAVAU, M.; JOANETTE, Y. Pragmatics, theory of mind and executive functions after a right-hemisphere lesion: Different patterns of deficits. Journal of Neurolinguistics, v. 22, n. 5, p. 413-426, 2009.

CHAMPAGNE-LAVAU, M.; JOANETTE, Y. Context processing during irony comprehension in right-frontal brain-damaged individuals. Clinical Linguistics \& Phonetics, v. 32, n. 8, p. 721-738, 2018.

CHAPEY, R. Aphasia, language intervention strategies in adult. 3. ed. Baltimore: Williams \& Wilkins, 1994.

DIAMOND, A. Executive functions. Annual review of psychology, v. 64, p. 135-168, 2013.

DIAZ, M. T.; EPPES, A. Factors influencing right hemisphere engagement during metaphor comprehension. Frontiers in Psychology, v. 9, p. 1-6, 28 mar. 2018. 
- | As bases neurais da linguagem e o hemisfério direito na construção de sentidos

DRONKERS, N. F.; PLAISANT, O.; IBA-ZIZEN, M. T.; CABANIS, E. A. Paul Broca's historic cases: High resolution MR imaging of the brains of Leborgne and Lelong. Brain, v. 130, n. 5, p. 1432-1441, 2007.

EVIATAR, Z.; JUST, M. A. Brain correlates of discourse processing: An fMRI investigation of irony and conventional metaphor comprehension. Neuropsychologia, v. 44, n. 12, p. 2348-2359, 2006.

FERSTL, E. C.; VON CRAMON, D. Y. What does the frontomedian cortex contribute to language processing: Coherence or theory of mind? Neurolmage, v. 17, n. 3, p. 1599-1612, 2002.

FRANÇA, A. I. Neurociência da linguagem. In: MAIA, M. (org.). Psicolinguística, psicolinguísticas: uma introdução. São Paulo: Editora Contexto, 2015. p. 171-188.

FRANKLIN, A.; CATHERWOOD, D.; ALVAREZ, J.; AXELSSON, E. Hemispheric asymmetries in categorical perception of orientation in infants and adults. Neuropsychologia, v. 48, n. 9, p. 2648-2657, jul. 2010.

GAJARDO-VIDAL, A.; LORCA-PULS, D. L.; HOPE, T. M. H.; PARKER JONES, O.; SEGHIER, M. L.; PREJAWA, S.; CRINION, J. T.; LEFF, A. P.; GREEN, D. W.; PRICE, C. J. How right hemisphere damage after stroke can impair speech comprehension. Brain, v. 141, n. 12, p. 3389-3404, 1 dez. 2018.

GERNSBACHER, M. A.; KASCHAK, M. P. Neuroimaging studies of language production and comprehension. Annual Review of Psychology, v. 54, n. 1, p. 91-114, 2003 a.

GERNSBACHER, M. A.; KASCHAK, M. P. Neuroimaging Studies of Language Production and Comprehension. Annual Review of Psychology, v. 54, n. 1, p. 91-114, fev. 2003b.

GIORA, R. Understanding figurative and literal language: The graded salience hypothesis. Cognitive Linguistics, v. 8, n. 3, p. 183-206, jan. 1997.

GIORA, R.; ZAIDEL, E.; SOROKER, N.; BATORI., G.; KASHER, A. Differential effects of right- and left-hemisphere damage on understanding sarcasm and metaphor. Metaphor and Symbol, v. 15, n. 1, p. 63-83, 2004. 
HICKOK, G.; SMALL, S. L. (ed.). The neurobiology of Language. London: Academic Press, 2016.

HOROWITZ-KRAUS, T.; WANG, Y.; PLANTE, E.; HOLLAND, S. Involvement of the right hemisphere in reading comprehension: A DTI study. Brain Research, v. 1582, p. 34-44, 2014.

HUTSLER, J.; GALUSKE, R. A. Hemispheric asymmetries in cerebral cortical networks. Trends Neurosci, v. 26, n. 8, p. 429-435, 2003.

INDEFREY, P. The spatial and temporal signatures of word production components. Cognition, v. 92, n. 1-2, p. 101-144, 2004.

JOHNS, C. L.; TOOLEY, K. M.; TRAXLER, M. J. Discourse impairments following right hemisphere brain damage: A critical review. Language and Linguistics Compass, v. 2, n. 6, p. 1038-1062, 2008.

JUNG-BEEMAN, M. Bilateral brain processes for comprehending natural language. Trends in Cognitive Sciences, v. 9, n. 11, p. 512-518, nov. 2005.

KAHLAOUI, K.; SCHERER, L. C.; JOANETTE, Y. The right hemisphere's contribution to the processing of semantic relationships between words. Language and Linguistics Compass, v. 2, n. 4, p. 550-568, 2008.

KANDEL, E. R.; SCHWARTZ, J. H.; JESSELL, T. H. A linguagem e as afasias. In: KANDEL, E. R.; SCHUWARTZ, J. H.; JESSEL, T. M. Princípios da neurociência. 4. ed. Barueri: Manole, 2003. cap. 59, p. 1169-1187.

KIDD, D. C.; CASTANO, E. Reading Literary Fiction Improves Theory of Mind. Science, v. 342, n. 6156, p. 377-380, 18 out. 2013.

KOTZ, S. A.; SCHWARTZE, M. Cortical speech processing unplugged: A timely subcorticocortical framework. Trends in Cognitive Sciences, v. 14, n. 9, p. 392-399, 2010.

KRISTENSEN, C. H.; ALMEIDA, R. M. M. de; GOMES, W. B. Desenvolvimento histórico e fundamentos metodológicos da neuropsicologia cognitiva. Psicologia: Reflexão e Crítica, v. 14, n. 2, p. 259-274, 2001. 
- | As bases neurais da linguagem e o hemisfério direito na construção de sentidos

KURCZEK, J.; DUFF, M. C. Intact discourse cohesion and coherence following bilateral ventromedial prefrontal cortex. Brain and Language, v. 123, n. 3, p. 222-227, 2012.

MALLOY-DINIZ, L. F.; FUENTES, D.; CONSENZA, R. M. Neuropsicologia do

Envelhecimento: uma abordagem multidimensional. Porto Alegre: Artmed, 2013.

MAR, R. A. The neuropsychology of narrative: story comprehension, story production and their interrelation. Neuropsychologia, v. 42, n. 10, p. 1414-1434, jan. 2004.

MARINI, A.; GALETTO, V.; ZAMPIERI, E.; VORANO, L.; ZETTIN, M.; CARLOMAGNO, S. Narrative language in traumatic brain injury. Neuropsychologia, v. 49, n. 10, p. 2904-2910, 2011.

MASON, R. A.; WILLIAMS, D. L.; KANA, R. K.; MINSHEW, N.; JUST, M. A. Theory of Mind disruption and recruitment of the right hemisphere during narrative comprehension in autism. Neuropsychologia, v. 46, n. 1, p. 269-280, 2008.

MASON, R. A.; JUST, M. A. Neuroimaging contributions to the understanding of discourse processes. In: TRAXLER, M.; GERNSBACHER, M. A. Handbook of Psycholinguistics. Academic Press, 2006. p. 765-799.

MORATO, E. M. Neurolinguística. In: MUSSALIN, F.; BENTES, A. C. (org.). Introdução à linguística: domínios e fronteiras. 8. ed. São Paulo: Cortez, 2012. p. 167-200.

NARAIN, C.; SCOTT, S. K.; WISE, R. J. S.; ROSEN, S.; LEFF, A.; IVERSEN, S. D.; MATTHEWS, P. M. Defining a left-lateralized response specific to intelligible speech using fMRI.

Cerebral Cortex, v. 13, n. 12, p. 1362-1368, 2003.

NEWMAN, S. D.; JUST, M. A.; MASON, R. Compreendendo o texto com o lado direito do cérebro - o que os estudos de neuroimagem funcional têm a dizer. In: RODRIGUES, C.; TOMITCH, L. M. B. (ed.). Linguagem e cérebro humano: contribuições multidisciplinares. Porto Alegre: Artmed, 2004.

OBLER, L. K.; RYKHLEVSKAIA, E.; SCHNYER, D.; CLARK-COTTON, M. R.; SPIRO, A. R.; HYUN, J.; KIM, D- S.; GORAL, M.; ALBERT, M. L. Bilateral brain regions associated with naming in older adults. Brain and Language, v. 113, n. 3, p. 113-123, 2010. 
OBLER, L. K.; CAPLAN, D. Neurolinguistics and Linguistic Aphasiology: An Introduction. Language, 1990.

ORTIZ, K. Z. (org.). Distúrbios neurológicos adquiridos: linguagem e cognição. 2. ed. Barueri: Manole, 2010.

PAROLA, A.; GABBATORE, I.; BOSCO, F. M.; BARA, B. G.; COSSA, F. M.; GINDRI, P.; SACCO, K. Assessment of pragmatic impairment in right hemisphere damage. Journal of Neurolinguistics, v. 39, p. 10-25, 2016.

POEPPEL, D.; EMMOREY, K.; HICKOK, G.; PYLKKANEN, L. Towards a New Neurobiology of Language. Journal of Neuroscience, v. 32, n. 41, p. 14125-14131, 2012.

PREMACK, D.; WOODRUFF, G. Does the chimpanzee have a theory of mind? Behavioral and Brain Sciences, v. 4, n. 1, p. 515-526, 1978.

PRICE, C. J. A review and synthesis of the first 20years of PET and fMRI studies of heard speech, spoken language and reading. Neurolmage, v. 62, n. 2, p. 816-847, 2012.

QI, Z.; LEGAULT, J. Neural hemispheric organization in successful adult language learning: Is left always right? Adult and Second Language Learning, v. 72, p. 119, 2020.

REDOLAR-RIPOLL, D. Neurociencia Cognitiva. Madrid: Editorial Médica Panamericana, 2014.

ROSSELLI, M.; ARDILA, A.; BERNAL, B. Modelo de conectividad de la circunvolución angular en el lenguaje: metaanálisis de neuroimágenes funcionales. Revista de Neurologia, v. 60, p. 495-503, 2015.

SAXE, R. Theory of Mind (Neural Basis). In: Encyclopedia of Consciousness. [s.I.] Elsevier, 2009. p. 401-409.

SAXE, R. Why and how to study Theory of Mind with fMRI. Brain Research, v. 1079, n. 1, p. 57-65, 2006.

SAXE, R.; MORAN, J. M.; SCHOLZ, J.; GABRIELI, J. Overlapping and non-overlapping brain regions for theory of mind and self reflection in individual subjects. Social cognitive and affective neuroscience, v. 1, n. 3, p. 229-234, 2006. 
- | As bases neurais da linguagem e o hemisfério direito na construção de sentidos

SCHERER, L. C. Como os hemisférios cerebrais processam o discurso: evidências de estudos comportamentais e de neuroimagem. In: CAMPOS, J. C.; PEREIRA, V. W. (ed.). Linguagem e cognição: relações interdisciplinares. Porto Alegre: EDIPUCRS, 2009. p. 77-102.

SCHMIDT, G. L.; SEGER, C. A. Neural correlates of metaphor processing: The roles of figurativeness, familiarity and difficulty. Brain and Cognition, v. 71, n. 3, p. 375-386, dez. 2009.

SHIBATA, M.; TOYOMURA, A.; ITOH, H.; ABE, J. Neural substrates of irony comprehension: A functional MRI study. Brain Research, v. 1308, p. 114-123, 2010.

SILAGI, M. L.; RADANOVIC, M.; CONFORTO, A. B.; MENDONÇA, L. I. Z.; MANSUR, L. L. Inference comprehension in text reading: Performance of individuals with right- versus left-hemisphere lesions and the influence of cognitive functions. PLoS ONE, v. 13, n. 5, p. e0197195, 2018.

ST GEORGE, M.; KUTAS, M.; MARTINEZ, A.; SERENO, M. I. Semantic integration in reading: engagement of the right hemisphere during discourse processing. Brain, v. 122, n. 7, p. 1317-1325, jul. 1999.

TOMITCH, L. M. B.; NEWMAN, D. S.; CARPENTER, P. A.; JUST, M. A. Main idea identification: a functional imaging study of a complex language comprehension process. In: TOMITCH, L. M. B.; RODRIGUES, C. (ed.). Ensaios sobre a linguagem e o cérebro humano: contribuiç̧ões multidisciplinares. Porto Alegre: Artmed, 2004. p. 167-175.

TREMBLAY, P.; DICK, A. S. Broca and Wernicke are dead, or moving past the classic model of language neurobiology. Brain and Language, v. 162, p. 60-71, 2016.

VIGNEAU, M.; BEAUCOUSIN, V.; HERVÉ, P. Y.; JOBARD, G.; PETIT, L.; CRIVELLO, F.; MELLET, E.; ZAGO, L.; MAZOYER, B.; TZOURIO-MAZOYER, N. What is right-hemisphere contribution to phonological, lexico-semantic, and sentence processing? Insights from a meta-analysis. Neurolmage, v. 54, n. 1, p. 577-593, 2011.

VIRTUE, S.; HABERMAN, J.; CLANCY, Z.; PARRISH, T.; JUNG BEEMAN, M. Neural activity of inferences during story comprehension. Brain Research, v. 1084, n. 1, p. 104-114, 2006.

WERNICKE, C. Some new studies on aphasia. In: ELING, P. (ed.). Reader in the history of aphasia: From Franz Gall to Norman Geschwind. v. 4. Philadelphia: John Benjamins Publishing, 1994. p. 69-98. (Trabalho original publicado em 1874). 
WIMMER, H.; PERNER, J. Beliefs about beliefs: Representation and constraining function of wrong beliefs in young children's understanding of deception. Cognition, v. 13, n. 1, p. 103-128, 1983.

YANG, J. The role of the right hemisphere in metaphor comprehension: A meta-analysis of functional magnetic resonance imaging studies. Human Brain Mapping, v. 35, n. 1, p. 107-122, jan. 2014.

YOUNG, L.; CUSHMAN, F.; HAUSER, M.; SAXE, R. The neural basis of the interaction between theory of mind and moral judgment. Proceedings of the National Academy of Sciences, v. 104, n. 20, p. 8235-8240, 15 maio 2007.

ZIV, M.; SMADJA, M.-L.; ARAM, D. Preschool teachers' reference to theory of mind topics in three storybook contexts: Reading, reconstruction and telling. Teaching and Teacher Education, v. 45, p. 14-24, jan. 2015.

COMO CITAR ESTE ARTIGO: SOUSA, Lucilene Bender de; SCHNEIDER, Fernanda. As bases neurais da linguagem e o hemisfério direito na construção de sentidos. Revista do GEL, v. 17, n. 3, p. 304-328, 2020. Disponível em: https:// revistadogel.gel.org.br/

DOI: http://dx.doi.org/10.21165/gel.v17i3.2864

Submetido em: 20/07/2020 | Aceito em: 02/09/2020. 